\title{
DOUBLE LAPLACE TRANSFORM METHOD FOR SOLVING TELEGRAPH EQUATION
}

\section{Muchammad Abrori ${ }^{*}$, Sugiyanto and Hana Mei Satriana Sari}

Department of Mathematics

Universitas Islam Negeri Sunan Kalijaga

Yogyakarta, Indonesia

e-mail: borymuch@yahoo.com

\begin{abstract}
Telegraph equation is a partial differential equation which includes the one dimensional wave equation. This equation can be used to solve several problems that physically regulate voltage and current in the electricity transmission line in distance and time. In this study, telegraph equation and partial integrodifferential equation were analyzed and resolved by double Laplace transform. This equation was accompanied by initial values and boundary values solved by Laplace transform. Next, there are some examples of equations to discover a solution. The solution of these examples showed that double Laplace transform is one method that can be used to solve telegraph equation. Then the solutions are simulated in graphical form which shows the waveform of the equation.
\end{abstract}

Received: March 12, 2019; Accepted: April 12, 2019

Keywords and phrases: telegraph equation, partial integrodifferential equation, double Laplace transform.

*Corresponding author 


\section{Introduction}

The telegraph equation is a differential partial equation developed by Oliver Heaviside in 1880 [1]. This equation included one wave dimensional equation (hyperbolic), which physically regulates the voltage and flow in the electricity transmission line with distance and time [2].

One application of telegraph equation is the solution of communication system problems involving the transmission of signals from one point to another [3]. So many mathematical methods can be used to obtain telegraph equation solutions, which is Laplace transform. Laplace transform is a method to solve homogeneous wave equations, whereas double Laplace transform of non-homogeneous equations [4].

Based on the description above, telegraph equations and partial integrodifferential equations will be solved by double Laplace transform. These equations are accompanied by initial values and boundary values, which are solved by Laplace transform.

The definitions are given by Debnath [5]. The double Laplace transform is defined by:

$$
\mathcal{L}_{x} \mathcal{L}_{t}[f(x, t)]=F(p, s)=\int_{0}^{\infty} e^{-p x} \int_{0}^{\infty} e^{-s t} f(x, t) d t d x
$$

where $x, t>0$ and $p, s$ are complex numbers.

Double Laplace transform for the first order partial derivative is defined as follows [6]:

$$
\mathcal{L}_{x} \mathcal{L}_{t}\left[\frac{\partial f(x, t)}{\partial t}\right]=s F(p, s)-F(p, 0)
$$

double Laplace transform for second order partial derivative with respect to $x$ is given by

$$
\mathcal{L}_{x x}\left[\frac{\partial^{2} f(x, t)}{\partial x^{2}}\right]=p^{2} F(p, s)-p F(0, s)-\frac{\partial F(0, s)}{\partial x},
$$


and double Laplace transforms for second partial derivative with respect to $t$ is given by

$$
\mathcal{L}_{t t}\left[\frac{\partial^{2} f(x, t)}{\partial t^{2}}\right]=s^{2} F(p, s)-s F(p, 0)-\frac{\partial F(p, 0)}{\partial t} .
$$

\section{Double Laplace Transform Analytic on Telegraph Equation}

There are so many problems in telegraph equation, for example, equation of Jang [2] as

$$
u_{x x}=u_{t t}+\alpha u_{t}+u-f(x, t)
$$

where $\alpha>0$ is a constant.

Equation (5) is then fitted to the initial value and the limit values are taken from Kashuri et al. [7]

$$
\begin{aligned}
& u(x, 0)=g_{1}(x), \quad u_{t}(x, 0)=g_{2}(x), \\
& u(0, t)=f_{1}(t), \quad u_{x}(0, t)=f_{2}(t) .
\end{aligned}
$$

Taking double Laplace to (5), we get

$$
\begin{aligned}
& \mathcal{L}_{x} \mathcal{L}_{t}\left[u_{x x}\right]=\mathcal{L}_{x} \mathcal{L}_{t}\left[u_{t t}+\alpha u_{t}+u-f(x, t)\right] \\
\Leftrightarrow & p^{2} U(p, s)-p U(0, s)-U_{x}(0, s)=\left(s^{2} U(p, s)-s U(p, 0)-U_{t}(p, 0)\right) \\
& +\alpha(s U(p, s)-U(p, 0))+U(p, s)-F(p, s) \\
\Leftrightarrow & \left(p^{2}-s^{2}-\alpha s-1\right) U(p, s)-p U(0, s)-U_{x}(0, s)+s U(p, 0) \\
& +U_{t}(p, 0)+\alpha U(p, 0)=-F(p, s),
\end{aligned}
$$

where $U(p, s)=\mathcal{L}_{x} \mathcal{L}_{t}[u(x, t)]$.

Taking single Laplace transform to initial and boundary values (6), we get

$$
\mathcal{L}[u(0, t)]=U(0, s)=F_{1}(s)
$$




$$
\begin{aligned}
& \mathcal{L}\left[u_{x}(0, t)\right]=U_{x}(0, s)=F_{2}(s), \\
& \mathcal{L}[u(x, 0)]=U(p, 0)=G_{1}(p), \\
& \mathcal{L}\left[u_{t}(x, 0)\right]=U_{t}(p, 0)=G_{2}(p) .
\end{aligned}
$$

By substituting (8) in (7), we obtain

$$
U(p, s)=\left(\frac{p F_{1}(s)+F_{2}(s)}{\left(p^{2}-s^{2}-\alpha s-1\right)}\right)-\left(\frac{s G_{1}(p)+G_{2}(p)+\alpha G_{1}(p)+F(p, s)}{\left(p^{2}-s^{2}-\alpha s-1\right)}\right)
$$

Applying inverse double Laplace transform to (9), we obtain the solution of (5) in the form

$$
\begin{aligned}
& u(x, t) \\
= & \mathcal{L}_{p}^{-1} \mathcal{L}_{s}^{-1}\left[\left(\frac{p F_{1}(s)+F_{2}(s)}{\left(p^{2}-s^{2}-\alpha s-1\right)}\right)-\left(\frac{s G_{1}(p)+G_{2}(p)+\alpha G_{1}(p)+F(p, s)}{\left(p^{2}-s^{2}-\alpha s-1\right)}\right)\right] .
\end{aligned}
$$

\section{Double Laplace Transform Analytic on Partial Integrodifferential Equation}

Integrodifferential equation is an integral equation containing the derivatives at the same time in it [8]. Consider the following general partial integrodifferential equation as in [9]:

$$
u_{t t}-u_{x x}+u+\int_{0}^{x} \int_{0}^{t} g(x-\alpha, t-\beta) u(\alpha, \beta) d \alpha d \beta=f(x, t),
$$

with the initial condition

$$
u(x, 0)=g_{1}(x), \quad u_{t}(x, 0)=g_{2}(x)
$$

and boundary conditions

$$
u(0, t)=f_{1}(t), \quad u_{x}(0, t)=f_{2}(t) .
$$


Taking double Laplace to (11), we get

$$
\begin{aligned}
& \mathcal{L}_{x} \mathcal{L}_{t}\left[u_{t t}-u_{x x}+u+\int_{0}^{x} \int_{0}^{t} g(x-\alpha, t-\beta) u(\alpha, \beta) d \alpha d \beta\right]=\mathcal{L}_{x} \mathcal{L}_{t}[f(x, t)] \\
\Leftrightarrow & \left(s^{2}-p^{2}+1+G(p, s)\right) U(p, s)+p U(0, s)+U_{x}(0, s) \\
& -s U(p, 0)-U_{t}(p, 0)=F(p, s) .
\end{aligned}
$$

Taking single Laplace transform to initial value (12) and boundary value (13), we get

$$
\begin{aligned}
& \mathcal{L}[u(0, t)]=U(0, s)=F_{1}(s), \\
& \mathcal{L}\left[u_{x}(0, t)\right]=U_{x}(0, s)=F_{2}(s), \\
& \mathcal{L}[u(x, 0)]=U(p, 0)=G_{1}(p), \\
& \mathcal{L}\left[u_{t}(x, 0)\right]=U_{t}(p, 0)=G_{2}(p) .
\end{aligned}
$$

By substituting (15) in (14), we obtain

$$
U(p, s)=\left(\frac{s G_{1}(p)+G_{2}(p)}{\left(s^{2}-p^{2}+1+G(p, s)\right)}\right)-\left(\frac{p F_{1}(s)+F_{2}(s)-F(p, s)}{\left(s^{2}-p^{2}+1+G(p, s)\right)}\right)
$$

Applying inverse double Laplace transform to (16), we obtain the solution of (11) in the form

$$
\begin{aligned}
& u(x, t) \\
= & \mathcal{L}_{p}^{-1} \mathcal{L}_{s}^{-1}\left[\left(\frac{s G_{1}(p)+G_{2}(p)}{\left(s^{2}-p^{2}+1+G(p, s)\right)}\right)-\left(\frac{p F_{1}(s)+F_{2}(s)-F(p, s)}{\left(s^{2}-p^{2}+1+G(p, s)\right)}\right)\right] .
\end{aligned}
$$

Example 1. In this case, the nonhomogeneous telegraph equation is given by

$$
u_{x x}=u_{t t}+u_{t}+u-6 e^{x+2 t}
$$

with the initial value

$$
u(x, 0)=e^{x}, \quad u_{t}(x, 0)=2 e^{x}
$$


and the limit value

$$
u(0, t)=e^{2 t}, \quad u_{x}(0, t)=e^{2 t}
$$

Taking double Laplace to (18), we get

$$
\begin{aligned}
& \mathcal{L}_{x} \mathcal{L}_{t}\left[u_{x x}\right]=\mathcal{L}_{x} \mathcal{L}_{t}\left[u_{t t}+u_{t}-u-6 x^{x+2 t}\right] \\
\Leftrightarrow & \left(p^{2}-s^{2}-s-1\right) U(p, s)=p U(0, s)+U_{x}(0, s)-s U(p, 0)-U_{t}(p, 0) \\
& -U(p, 0)-\frac{6}{(p-1)(s-2)} .
\end{aligned}
$$

Taking single Laplace transform to initial value (19) and boundary value (20), we get

$$
\begin{aligned}
& \mathcal{L}[u(0, t)]=U(0, s)=\frac{1}{(s-2)}, \\
& \mathcal{L}\left[u_{x}(0, t)\right]=U_{x}(0, s)=\frac{1}{(s-2)}, \\
& \mathcal{L}[u(x, 0)]=U(p, 0)=\frac{1}{(p-1)}, \\
& \mathcal{L}\left[u_{t}(x, 0)\right]=U_{t}(p, 0)=\frac{2}{(p-1)}
\end{aligned}
$$

By substituting (22) in (21), we obtain

$$
U(p, s)=\left(\frac{\left(p^{2}-s^{2}-s-1\right)}{(p-1)(s-2)}\right)\left(\frac{1}{\left(p^{2}-s^{2}-s-1\right)}\right) .
$$

Applying inverse double Laplace transform to (23), we obtain the solution of (18) in the form

$$
u(x, t)=\mathcal{L}_{p}^{-1} \mathcal{L}_{s}^{-1}\left[\frac{1}{(p-1)(s-2)}\right]=e^{x+2 t} .
$$

The exact solution of telegraph equation is $u(x, t)=e^{x+2 t}$. 
We put domain of distance $0 \leq x \leq 5$ and domain of time $0 \leq t \leq 5$ on the exact solution of this telegraph equation.

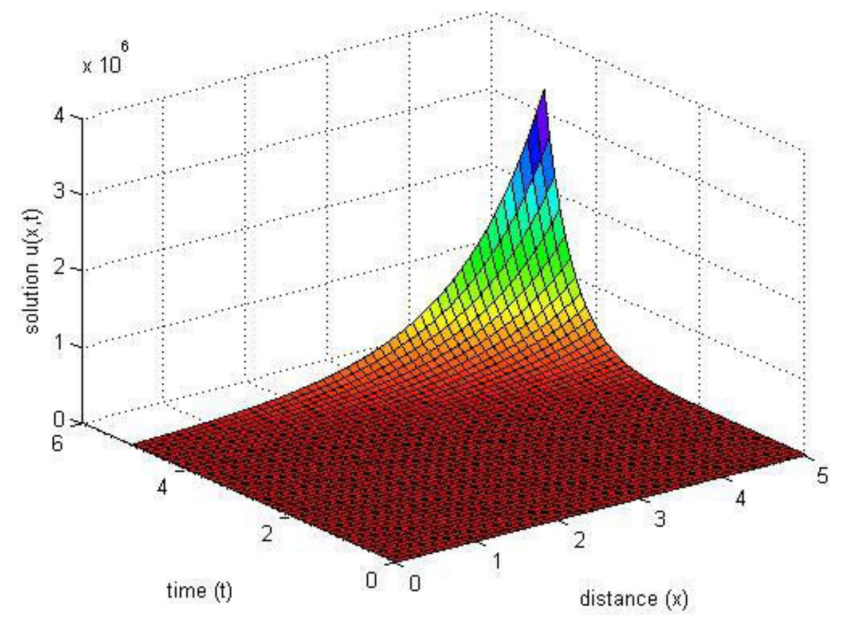

Figure 1

Figure 1 shows that peak points of domain $0 \leq x \leq 5$ and domain of time $0 \leq t \leq 5$ are, respectively, at $x=5$ and $t=5$.

Example 2. In this case, the nonhomogeneous telegraph equation is given by

$$
u_{x x}=u_{t t}+3 u_{t}+u-6 e^{t} \sin x
$$

with the initial value

$$
u(x, 0)=\sin x, \quad u_{t}(x, 0)=\sin x,
$$

and the limit value

$$
u(0, t)=0, \quad u_{x}(0, t)=e^{t}
$$

Taking double Laplace to (25), we get

$$
\begin{aligned}
& \mathcal{L}_{x} \mathcal{L}_{t}\left[u_{x x}\right]=\mathcal{L}_{x} \mathcal{L}_{t}\left[u_{t t}+3 u_{t}+u-6 e^{t} \sin x\right] \\
\Leftrightarrow & \left(p^{2}-s^{2}-3 s-1\right) U(p, s)=p U(0, s)+U_{x}(0, s)-s U(p, 0)-U_{t}(p, 0) \\
& -3 U(p, 0)-\frac{6}{\left(p^{2}+1\right)(s-1)} .
\end{aligned}
$$


Taking single Laplace transform to initial value (26) and boundary value (27), we get

$$
\begin{aligned}
& \mathcal{L}[u(0, t)]=U(0, s)=0, \\
& \mathcal{L}\left[u_{x}(0, t)\right]=U_{x}(0, s)=\frac{1}{(s-1)}, \\
& \mathcal{L}[u(x, 0)]=U(p, 0)=\frac{1}{\left(p^{2}+1\right)}, \\
& \mathcal{L}\left[u_{t}(x, 0)\right]=U_{t}(p, 0)=\frac{1}{\left(p^{2}+1\right)} .
\end{aligned}
$$

By substituting (29) in (28), we obtain

$$
U(p, s)=\left(\frac{\left(p^{2}-s^{2}-3 s-1\right)}{(s-1)\left(p^{2}+1\right)}\right)\left(\frac{1}{\left(p^{2}-s^{2}-3 s-1\right)}\right) .
$$

Applying inverse double Laplace transform (30), we obtain the solution of (25) in the form

$$
u(x, t)=\mathcal{L}_{p}^{-1} \mathcal{L}_{s}^{-1}\left[\frac{1}{(s-1)\left(p^{2}+1\right)}\right]=e^{t} \sin x .
$$

The exact solution of standard telegraph equation is $u(x, t)=e^{t} \sin x$.

We put domain of distance $0 \leq x \leq 10$ and domain of time $0 \leq t \leq 10$ on the exact solution of this telegraph equation.

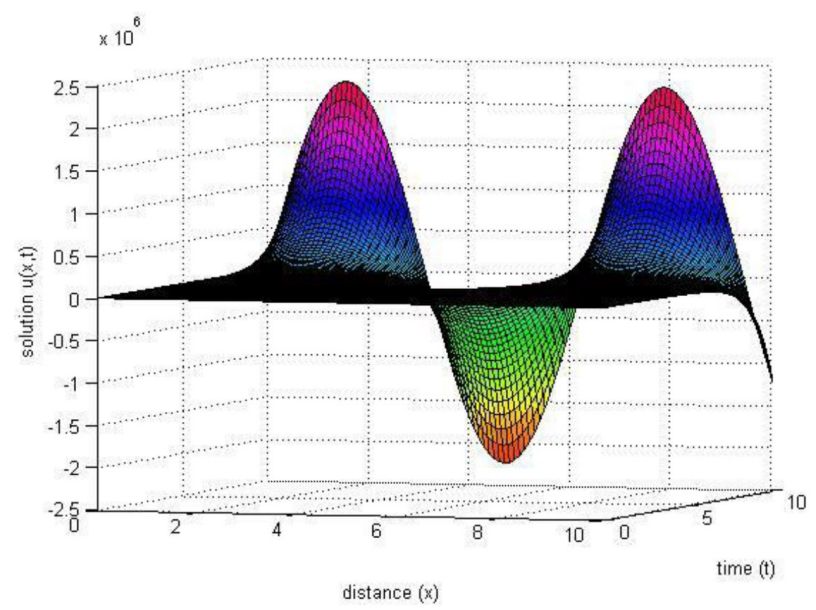

Figure 2 
Double Laplace Transform Method for Solving Telegraph ...

Figure 2 shows that domain $0 \leq x \leq 10$ and domain of time $0 \leq t \leq 10$, waves from the equation solution move up and down.

Example 3. In this case, partial integrodifferential equation is given by

$$
u_{t t}-u_{x x}+u+2 \int_{0}^{x} \int_{0}^{t} e^{x-\alpha+t-\beta} u(\alpha, \beta) d \alpha d \beta=e^{x+t}+2 x t e^{x+t}
$$

with the initial value and the limit value

$$
u(x, 0)=e^{x}, u_{t}(x, 0)=e^{x}, u(0, t)=e^{t}, u_{x}(0, t)=e^{t} .
$$

Taking double Laplace to (32), we get

$$
\begin{aligned}
& \mathcal{L}_{x} \mathcal{L}_{t}\left[u_{t t}-u_{x x}+u+2 \int_{0}^{x} \int_{0}^{t} e^{x-\alpha+t-\beta} u(\alpha, \beta) d \alpha d \beta\right] \\
= & \mathcal{L}_{x} \mathcal{L}_{t}\left[e^{x+t}+2 x t e^{x+t}\right] \\
\Leftrightarrow & \left(s^{2}-p^{2}+1+\frac{2}{(p-1)(s-1)}\right) U(p, s) \\
= & s U(p, 0)+U_{t}(p, 0)-p U(0, s)-U_{x}(0, s) \\
& +\frac{1}{(p-1)(s-1)}+\frac{2}{(p-1)^{2}(s-1)^{2}}
\end{aligned}
$$

Taking single Laplace transform to initial and boundary value (33), we get

$$
\begin{aligned}
& \mathcal{L}[u(0, t)]=U(0, s)=\frac{1}{(s-1)}, \\
& \mathcal{L}\left[u_{x}(0, t)\right]=U_{x}(0, s)=\frac{1}{(s-1)}, \\
& \mathcal{L}[u(x, 0)]=U(p, 0)=\frac{1}{(p-1)}, \\
& \mathcal{L}\left[u_{t}(x, 0)\right]=U_{t}(p, 0)=\frac{1}{(p-1)}
\end{aligned}
$$


274 Muchammad Abrori, Sugiyanto and Hana Mei Satriana Sari

By substituting (35) in (34), we obtain

$$
U(p, s)=\left(\frac{\left(s^{2}-p^{2}+1\right)(p-1)(s-1)+2}{(p-1)^{2}(s-1)^{2}}\right)\left(\frac{(p-1)(s-1)}{\left(s^{2}-p^{2}+1\right)(p-1)(s-1)+2}\right)
$$

Applying inverse double Laplace transform to (36), we obtain the solution of (32) in the form

$$
u(x, t)=\mathcal{L}_{p}^{-1} \mathcal{L}_{s}^{-1}\left[\frac{1}{(p-1)(s-1)}\right]=e^{x+t} .
$$

The exact solution of standard partial integrodifferential equation is $u(x, t)=e^{x+t}$.

We put domain of distance $1 \leq x \leq 3$ and domain of time $2 \leq t \leq 4$ on the exact solution of this telegraph equation

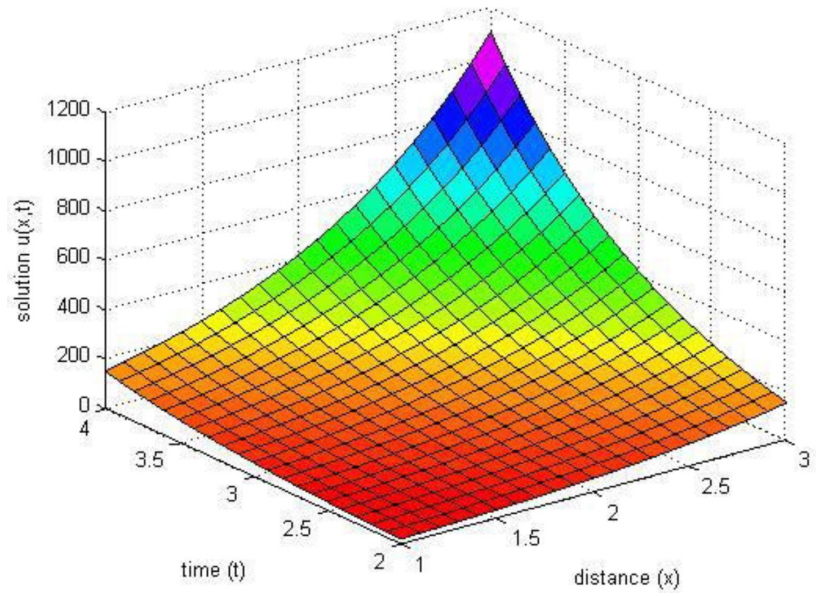

Figure 3

Figure 3 shows that peak points of domain $1 \leq x \leq 3$ and domain of time $2 \leq t \leq 4$ are, respectively, at $x=3$ and $t=4$. 


\section{Conclusion}

We have applied double Laplace transform to obtain exact solution of telegraph equation and partial integrodifferential equation. The solutions of the equations are simulated in graphical form which shows the waveform of the equation.

\section{References}

[1] Ranjit R. Dhunde and G. L. Waghmare, Double Laplace transform method for solving space and time fractional telegraph equation, Int. J. Math. Math. Sci. 2016, Art. ID 1414595, 7 pp.

[2] T. S. Jang, A new solution procedure for the nonlinear telegraph equation, Commun. Nonlinear Sci. Numer. Simul. 29(1-3) (2015), 307-326.

[3] P. Veeresha and D. G. Prakasha, Numerical solution for fractional model of telegraph equation by using $q$-HATM, India, 2018.

arXiv preprint arXiv:1805.03968.

[4] Hassan Eltayeb and Adem Kiliçman, A note on the solution of wave, Laplace's and heat equations with convolution terms by using a double Laplace transform, Appl. Math. Lett. 21(12) (2008), 1324-1329.

[5] Lokenath Debnath, The double Laplace transforms and their properties with applications to functional, integral and partial differential equations, Int. J. Appl. Comput. Math. 2(2) (2016), 223-241.

[6] Adem Kiliçman and Hassan Eltayeb, A note on defining singular integral as distribution and partial differential equations with convolution term, Math. Comput. Modelling 49(1-2) (2009), 327-336.

[7] Artion Kashuri, A. Fundo and R. Liko, On double new integral transform and double Laplace transform, European Scientific Journal, ESJ 9(33) (2013), 82-90.

[8] Abdul-Majid Wazwaz, Volterra integro-differential equations, Linear and Nonlinear Integral Equations, Springer, Berlin, Heidelberg, 2011.

[9] Hassan Eltayeb and Adem Kiliçman, A note on double Laplace transform and telegraphic equation, Abstr. Appl. Anal. 2013, Art. ID 932578, 6 pp. 